\title{
3 Bewegung und Ernährungsprogramme
}

\author{
Claudia Sellschopp und Willi Heepe
}

\subsection{Ernährung und Männergesundheit}

Kein Land der Welt hat so viele Ernährungsangebote in Sachen Gesundheit wie Deutschland. Die Anzahl der vermarkteten Diäten erreicht die Zahl dreihundert. Gemeinsam haben sie nicht verhindern können, dass Deutschland sowohl bei den Männern als auch bei den Frauen Europameister im Übergewicht geworden ist. Eine Analyse des Versagens liegt nicht vor. Solide Daten für positive Auswirkungen auf die Gesundheit liegen bei verschiedenen chronischen Krankheiten, wie zum Beispiel Diabetes mellitus, Fettstoffwechselstörungen etc. vor. Die Erfolgschancen im Bereich Adipositas sind näherungsweise so frustrierend wie die Therapie der Alkoholkrankheit. Für die koronare Herzerkrankung und generell die Arteriosklerose liegen ebenfalls solide Daten vor. Zum Thema Männergesundheit und Ernährung existiert wenig Konkretes. Worin begründet sich dieser Zustand?

Der gemeinsame Nenner aller Ernährungsempfehlungen liegt in der Bevorzugung besonders gesundheitsfördernd bewerteter Nahrungskomponenten. Gezeigt werden Obstteller, Milch- und Quarkspeisen, magere Käse-, Wurst- und Fleischvarianten. Auch die Getreidepalette ist ausreichend vertreten. Die Argumentationskette ist überzeugend, die Erfolge ernüchternd. Welche Faktoren werden nicht genügend gewertet und gewürdigt?

- Das Bewegungsaufkommen des Menschen hat sich in einer Generation von historisch 8-12 h auf circa 20-25 min täglich reduziert (DSH-Köln). Eine Anpassung der Ernährung dieser Tatsache folgend hat nicht stattgefunden. Die Alternative, das Bewegungsaufkommen an die Ernährung anzupassen, hat ebenfalls nicht stattgefunden. Wer über Ernährung redet und die Bewegung ausblendet, weiß nicht wovon er redet. Jeder Körpertyp repräsentiert ein eigenes psychophysisches, sehr komplexes Modell und bedarf einer Beratung unter sorgfältiger Berücksichtigung aller Komponenten. 
- Die Nahrungsdichte hat enorm zugenommen. Unendlich viele Lebensmittel und Getränke beinhalten mit geringem Volumen eine extrem hohe Energiedichte. Sie sind die entscheidenden Dienstleister in Sachen Übergewicht.

- Verbrachten die Menschen früher viel Zeit mit dem Zubereiten und Verzehren der Nahrung, so ist heute die Essenszeit für die Mehrzahl der Menschen auf wenige Minuten zusammengeschrumpft. Da die Sättigung ein sich langsam entwickelnder Prozess ist, beinhaltet jedes schnelle Essen die Gefahr zu viel zu essen und liefert ebenfalls einen Baustein zum Übergewicht.

- Der Nachkriegsgeneration wurde ein weiteres fatales Verhaltensmuster anerzogen. Aus der Nachkriegsnot war die Verschwendung von Nahrung eine Todsünde. Eine bewusste Erziehung oder Hinlenkung, bei sich einstellendem Sättigungsgefühl die Nahrungsaufnahme auch bei noch halb vollem Teller zu beenden, wurde nie geübt.

- Ein weiteres Übel liegt in der Tatsache, dass beinahe alle angebotenen Nahrungsmittel mit Geschmackskorrigentien oder -verstärkern versehen werden und damit ein natürliches Geschmackserleben und eine Kultivierung von Essen und Trinken systematisch gestört werden.

- Die Aufbaujahre nach 1945 bedingten, mangels Maschinen, hohe Arbeitsleistungen mit hohem Energiebedarf. Gleichzeitig waren die Menschen aus der Kriegszeit ausgehungert. Die Problemlösung lag in einer fettreichen und quantitativ voluminösen Essensweise. Mit dem Arbeitstrukturwandel begann die Ära des Übergewichts. Die sich dann einstellende Fluchtwelle in die Kohlenhydrate verschlimmerte die Situation noch mehr und löste über den sich einstellenden Hyperinsulinismus die Flutwelle Diabetes mellitus Typ II aus. Viele Menschen leben heute in einem physiologischen Defizit dahingehend, dass sie durch ihre Tätigkeit wesentlich weniger Arbeitskalorien verbrennen, als für den Körpertyp von der Natur vorgesehen. Essen sie, was ihr Körper wünscht, werden sie übergewichtig. Essen sie, was sie wirklich verbrennen, sind sie ständig eher hungrig.

Ernährung und Bewegung gehören in jede Diskussion über gesunde Lebensweisen. Ausgeblendet werden häufig dabei das sinnliche Element von Essen und Trinken, der hohe Genusswert und die Paraerotik. Aus der Sicht der mentalen Medizin sind die letzteren Punkte in ihrer Wertigkeit höher einzuschätzen als eine nach ausschließlichen Gesundheitskriterien zusammengesetzte Ernährung.

\section{Empfehlungen}

1. Zeit nehmen zum Essen! Sättigung ist ein langsamer Prozess. Schnellesser essen immer zu viel.

2. Die Nahrungskomponenten sinnvoll über den Tag verteilen. Zum Beispiel langkettige Kohlenhydrate wie Getreideprodukte am Morgen geben Energie bis zum Mittag. Eiweiße, Mineralien, Vitamine etc. geschickt verteilen bis zum Abend. Keine Spätmahlzeit. Die letzte Mahlzeit nach Genusswert selektiert, optisch exzellent aufbereitet, bewusst langsam erleben. Ein gutes Glas Wein dazu erhöht den Genuss und ist gesundheitlich äußerst positiv zu bewerten insbesondere auch auf die sexuelle Gesundheit. 


\subsection{Sport und Männergesundheit}

Der Mensch bewegt sich in Deutschland im Mittel noch circa 20-25 min auf eigenen Beinen. Im Gegenzug liegt die tägliche Fernsehzeit bei näherungsweise 5 h. Die Folgen sind sichtbar. Näherungsweise sind circa 70\% der Männer übergewichtig, die Hälfte davon adipös. Diese Immobilität beeinträchtigt sämtliche Körperfunktionen und hat katastrophale Auswirkungen auf die Gesundheit. Beinahe jeder Adipöse wird Diabetiker Typ II und legte damit den Grundstein für eine schwer therapierbare erektile Dysfunktion. Des Weiteren hat die Immobilität sehr viele Einflüsse nicht nur auf das Celenk- und Muskelsystem, sondern auch auf den Hormonhaushalt. So führt Trainingsmangel, d.h. Bewegungsmangel, genauso wie Übertraining zu einem deutlichen Testosteronmangel. Auf der anderen Seite ist eine ausgeglichene Bewegungsbilanz die Grundlage eines gesunden Hormonstatus. Sport- und Bewegungsempfehlungen gehören zu jeder Gesundheitsberatung. Sie sollten sich begründen auf dem Boden einer soliden Leistungsdiagnostik und alle bedingenden Faktoren berücksichtigen. Effektive Programme sind immer individuell; jeder pauschalierte Ansatz ist frustran.

Der Schwerpunkt dieser Empfehlungen liegt in der Gestaltung eines moderaten Ausdauertrainings mit dem Ziel die Herz-Kreislaufleistungsbreite altersadäquat zu entwickeln und langjährig bis zum Lebensende zu erhalten. Ein gut trainiertes Herz führt zu einer niedrigeren Pulsfrequenz, einem niedrigen Blutdruck und einer parasympathikotonen Gesamtregulation. Sie ist die absolute Bedingung für eine ruhigere, gelassenere und entspanntere Lebensweise. Die meisten Menschen brauchen hierzu heute schon Pharmaka wie Betarezeptorenblocker oder Psychopharmaka. Alle diese Pharmagruppen haben fatale Wirkungen auf die Vita sexualis und die gesamte Lebensdynamik. Unstreitig ist, dass der katastrophale Bewegungsmangel eine nie dagewesene Dimension erreicht hat. Beinahe jede Zivilisationskrankheit begründet sich schwerpunktmäßig in diesem Mangel und wird durch ihn verstärkt. Bewegungsmangel ist ein in sich progressiver Prozess und beschleunigt sich durch jedes Kilogramm Übergewicht und jede degenerierte Muskelfaser. Zusätzlich wird zu jeder körperlichen Bewegung ein erhöhtes sympathikotones Aktionspotenzial erforderlich. Letzteres beinhaltet auch die Gefahr von Gefäßspasmen mit dem Schwerpunkt einer vasospastischen Angina Pectoris und äußert negative Auswirkungen auf die Sexualität. Diese negativen Prozesse optimieren sich durch einen global hohen Leistungsdruck unter dem beinahe jeder Berufstätige in dieser Gesellschaft leidet. Wiederum gilt, Sport und Bewegungsberatung müssen individuell erfolgen, eine moderne umfassende Leistungsdiagnostik optimiert diese Beratung. Gerade bei der erektilen Dysfunktion kommt diesem Segment eine besondere Bedeutung zu. Neben der allgemeinen Fitness mit dem Schwerpunkt kardiale Fitness steht bei der erektilen Dysfunktion der Beckenboden im Mittelpunkt der Betrachtung. Ein optimaler Beckenboden sollte elastisch, kraftvoll, flexibel und schmerzfrei sein. Auf ihm ruht und lastet die Wirbelsäule mit der komplexen Problematik des gesamten Oberkörpers. Ebenso ist er die Brücke zu den unteren Extremitäten. Letztere sind häufig durch extreme Immobilität massiv degeneriert und in ihrer Muskulatur atroph. In der modernen Fitnesswelt wird eher der sichtbare Muskelanteil wie Bizeps etc. trainiert, der Beckenboden ist Tabuzone. Die tragende Basis dieses Bereiches muss vermittelt werden. Übungsprogramme müssen immer in Gesamtkonzepte integriert werden. Über Wahrnehmungsübungen müssen Tabus aufgebrochen werden. Das bewusste Wahrneh- 
men dieser wichtigen Rolle des Beckenbodens stärkt nicht nur das positive Körpergefühl sondern auch das Selbstwertgefühl und ist ein Stück Basis für eine gesunde Sexualität. Gezielte Übungsprogramme werden in zahlreichen Büchern, Skripten und Broschüren angeboten. Im Tenor sind sie gleich und beinahe immer akzeptabel. Die Problematik liegt in einer konsequenten Durchführung von Übungsprogrammen. In Eigenregie, selbst erarbeitet oder nicht kompetent angeleitet, schlafen solche Programme immer wieder ein und sind wenig und nicht dauerhaft erfolgreich. Nach eigenen Erfahrungen ist eine professionell geführte Gruppe 8-15 Männer) unter Anleitung eines fachlich gut ausgebildeten Trainers, mit hervorragendem Einfühlungsvermögen als optimal und erfolgversprechend anzusehen. Geduld, Ausdauer und das Wissen, dass der Erfolg Zeit braucht, müssen vermittelt werden. Die Übungen müssen mindestens zwei bis dreimal pro Woche durchgeführt werden. Ein Erfolg ist in Monaten zu erwarten.

\section{Literatur}

Franklin E (2002) Beckenbodenpower: Das dynamische Training für Sie und Ihn. Deutsche Ausgabe. Kösel-Verlag München

Seleger M, Krucker J, Keller Y, Trinkler F (2005) Die versteckte Kraft im Mann: Eine Anleitung zu mehr Lebensqualität und Potenz. BeBo Velag GmbH Zürich 